\title{
Cosine similarity measures for dual hesitant fuzzy sets
}

\author{
Wei Qiu ${ }^{1,2}$ a , Guangchun Zhang ${ }^{2, b}$ and Lei Zhu ${ }^{2, c}$ \\ ${ }^{1}$ Chinese Satellite Maritime Tracking and Controlling Department, Jiangyin 214431, China; \\ 2 PLA University of Science and Technology,Nanjing 210007, China. \\ a'qiuweinj@126.com, ${ }^{b}$ zhanggcnj@126.com, ${ }^{\mathrm{c}}$ zhuleinj@yeah.net
}

Keywords: similarity measures, hesitant fuzzy set.

\begin{abstract}
The Dual Fuzzy Sets (DHFSs), originated by Zhu and Xu [1], is a useful tool to deal with vagueness and ambiguity. In this paper, we propose one new measure called Cosine similarity measure for dual hesitant fuzzy sets, based on which the corresponding similarity measures can be obtained. We investigate the connections of the aforementioned distance measures and further develop a number of dual hesitant ordered weighted distance measures and dual hesitant ordered weighted similarity measures. They can alleviate the influence of unduly large (or small) deviations on the aggregation results by assigning them low (or high) weights. Several numerical examples are provided to illustrate these distance and similarity measures.
\end{abstract}

\section{Introduction}

Similarity measures of FSs are an important research topic in the FS theory [2], which has received much attention from researchers. For example, $\mathrm{Xu}$ [6] introduced the concepts of deviation degrees and similarity degrees between two linguistic values, and between two linguistic preference relations, respectively. Li and Cheng [7] generalized the Hamming distance and the Euclidean distance by adding a parameter and gave a similarity formula for IFSs only based on the membership degrees and non-membership degrees. Hung and Yang [8] and Grzegorzewski[9] suggested a lot of similarity measures for IFSs and interval-valued fuzzy sets based on the Hausdorff metric. Xu and Xia [10] proposed a variety of distance measures for hesitant fuzzy sets. They investigated the connections of the distance measures and further developed a number of hesitant ordered weighted distance measures.

Recently, Zhu and Xu [1] introduced the definition of dual hesitant fuzzy set. Dual hesitant fuzzy set can reflect the human's hesitance more objectively than the other classical extensions of fuzzy set ((intuitionistic fuzzy set [3], hesitant fuzzy set [4, 5], etc.)), thus it is very necessary to develop some theories about dual hesitant fuzzy sets. However, little has been done about this issue. The aforementioned measures, however, cannot be used to deal with the distance and similarity between DHFSs. Due to the fact that hesitancy is a very common problem in decision making as mentioned earlier, it is necessary to develop some measures for DHFSs. To do this, the remainder of the paper is organized as follows. Section 2 aims to presents the axioms for distance and the similarity measures. Finally, Section 3 gives the concluding remarks.

\section{Cosine similarity measure for DHFSs}

Definition 1[1]. Let $X$ be a fixed set, then a dual hesitant fuzzy set (DHFS) $D$ on $X$ is described as:

$$
D=\{<x, h(x), g(x)>\mid x \in X\}
$$

in which $h(x)$ and $g(x)$ are two sets of some values in [0,1], denoting the possible membership degrees and non-membership degrees of the element $x \in X$ to the set $D$ respectively, with the conditions:

$$
0 \leq \gamma, \eta \leq 1,0 \leq \gamma^{+}+\eta^{+} \leq 1
$$


where $\gamma \in h(x), \eta \in g(x), \gamma^{+} \in h^{+}(x)=\bigcup_{\gamma \in h(x)} \max \{\gamma\}$, and $\eta^{+} \in g^{+}(x)=\bigcup_{\eta \in g(x)} \max \{\eta\}$ for all $x \in X$. For convenience, the pair $d_{E}(x)=\left(h_{E}(x), g_{E}(x)\right)$ is called a dual hesitant fuzzy element (DHFE) denoted by $d=(h, g)$, with the conditions: $\gamma \in h(x), \eta \in g(x), \gamma^{+} \in h^{+}(x)=\bigcup_{\gamma \in h(x)} \max \{\gamma\}$, and $\eta^{+} \in g^{+}(x)=\bigcup_{\eta \in g(x)} \max \{\eta\}$, $0 \leq \gamma, \eta \leq 1$ and $0 \leq \gamma^{+}+\eta^{+} \leq 1$.

Definition 2 [6]. Let $A$ and $B$ be two DHFSs on $X=\left\{x_{1}, x_{2}, \ldots x_{n}\right\}$, then the similarity measure between $A$ and $B$ is defined as $s(A, B)$, which satisfy the following properties:

$$
\begin{aligned}
& \text { 1) } 0 \leq s(A, B) \leq 1 ; \\
& \text { 2) } s(A, B)=1 \text { if onlyif } A=B ; \\
& \text { 3) } s(A, B)=s(B, A) .
\end{aligned}
$$

To operate correctly, we should extend the shorter one until both of them have the same length when we compare them. In [11], Xu and Xia extended the shorter one by adding different values in hesitant fuzzy environments. Assume that there are two DHFSs $A$ and $B$ on $X=\left\{x_{1}, x_{2}, \ldots x_{n}\right\}$.Based on the extension of the cosine measure for fuzzy sets, a cosine similarity measure between DHFSs $A$ and $B$ is proposed as follows:

$$
\begin{gathered}
S_{1}(A, B)=\sum_{i=1}^{n} \frac{1}{n} \frac{\sum_{j=1}^{\# h_{x_{i}}} \gamma_{A}^{\sigma(j)}\left(x_{i}\right) \gamma_{B}^{\sigma(j)}\left(x_{i}\right)+\sum_{k=1}^{\# g_{x_{i}}} \eta_{A}^{\sigma(k)}\left(x_{i}\right) \eta_{B}^{\sigma(k)}\left(x_{i}\right)}{\sqrt{\sum_{j=1}^{\# h_{x_{i}}}\left(\gamma_{A}^{\sigma(j)}\left(x_{i}\right)\right)^{2}+\sum_{k=1}^{\# g_{x_{i}}}\left(\eta_{A}^{\sigma(k)}\left(x_{i}\right)\right)^{2}} \sqrt{\sum_{j=1}^{\# h_{x_{i}}}\left(\gamma_{B}^{\sigma(j)}\left(x_{i}\right)\right)^{2}+\sum_{k=1}^{\# g_{x_{i}}}\left(\eta_{B}^{\sigma(k)}\left(x_{i}\right)\right)^{2}}} \\
S_{2}(A, B)=\sum_{i=1}^{n} \frac{1}{n} \frac{\sum_{j=1}^{\# h_{x_{i}}} \gamma_{A}^{\sigma(j)}\left(x_{i}\right) \gamma_{B}^{\sigma(j)}\left(x_{i}\right)+\sum_{k=1}^{\# g_{x_{j}}} \eta_{A}^{\sigma(k)}\left(x_{i}\right) \eta_{B}^{\sigma(k)}\left(x_{i}\right)}{\max \left\{\left(\sum_{j=1}^{\# h_{x_{i}}}\left(\gamma_{A}^{\sigma(j)}\left(x_{i}\right)\right)^{2}+\sum_{k=1}^{\# g_{x_{i}}}\left(\eta_{A}^{\sigma(k)}\left(x_{i}\right)\right)^{2}\right),\left(\sum_{j=1}^{\# h_{x_{i}}}\left(\gamma_{B}^{\sigma(j)}\left(x_{i}\right)\right)^{2}+\sum_{k=1}^{\# g_{x_{i}}}\left(\eta_{B}^{\sigma(k)}\left(x_{i}\right)\right)^{2}\right)\right\}}
\end{gathered}
$$

In what follows, we give an example adapted from [17] to illustrate our distance measures for DHFSs.Example. Energy is an indispensable factor for the socio-economic development of societies. Thus the correct energy policy affects economic development and environment, and so, the most appropriate energy policy selection is very important. Suppose that there are five alternatives (energy projects) $Y_{i}(i=1,2,3,4,5)$ to be invested, and four attributes to be considered: $G_{1}$ : technological; $G_{2}$ : environmental; $G_{3}$ : socio-political; $G_{4}$ : economic. The attribute weight vector is $w=(0.15,0.3,0.2,0.35)^{T}$. The DHFS is just a tool to deal with such cases, and all possible evaluations for an alternative under the attributes can be considered as a DHFS. The results evaluated by the decision makers are contained in a hesitant fuzzy decision matrix, shown in Table 1. 
Table 1 Hesitant fuzzy decision matrix.

\begin{tabular}{ccccc}
\hline & $G_{1}$ & $G_{2}$ & $G_{3}$ & $G_{4}$ \\
\hline$Y_{1}$ & $\{(0.5, .04,0.3) ;(0.4,0.2)\}$ & $\{(0.9,0.8,0.7,0.1) ;(0.1,0)\}$ & $\{(0.5, .04,0.2) ;(0.5,0.3,0.1)\}$ & $\{(0.9,0.6,0.5,0.3) ;(0.1)\}$ \\
$Y_{2}$ & $\{(0.5,0.3) ;(0.5,0.4,0.1)\}$ & $\{(0.9,0.7,0.6,0.5,0.2) ;(0.1)\}$ & $\{(0.8,0.6,0.5,0.1) ;(0.2,0.1)\}$ & $\{(0.7,0.4,0.3) ;(0.2,0)\}$ \\
$Y_{3}$ & $\{(0.7,0.6) ;(0.2)\}$ & $\{(0.9,0.6) ;(0)\}$ & $\{(0.7,0.5,0.3) ;(0.3,0.2)\}$ & $\{(0.6,0.4) ;(0.3,0.1)\}$ \\
$Y_{4}$ & $\{(0.8,0.7,0.4,0.3) ;(0.2,0.1)\}$ & $\{(0.7,0.4,0.2) ;(0.3,0.2)\}$ & $\{(0.8,0.1) ;(0.1)\}$ & $\{(0.9,0.8,0.6) ;(0.1,0)\}$ \\
$Y_{5}$ & $\{(0.9,0.7,0.6,0.3,0.1) ;(0.1)\}$ & $\{(0.8,0.7,0.6,0.4) ;(0.2,0)\}$ & $\{(0.9,0.8,0.7) ;(0)\}$ & $\{(0.9,0.7,0.6,0.3) ;(0.1)\}$ \\
\hline
\end{tabular}

Suppose that the ideal alternative is $A=\{\{(1)\},\{0\}\}$ seen as a special DHFS, we can calculate the distance between each alternative and the ideal alternative using our distance measures.

If we use the generalized dual hesitant weighted distance and the generalized dual hesitant Hausdorff distance to calculate the deviations between each alternative and the ideal alternative, then we get the rankings of these alternatives, which are listed in Tables $2-3$, respectively, when some values of the parameter are given. We find that the rankings are different as the parameter (which can be considered as the decision makers' risk attitude) changes, consequently, the proposed distance measures can provide the decision makers more choices as the different values of the parameter are given according to the decision makers’ risk attitudes.

Table 2 Results obtained by the generalized dual hesitant weighted distance.

\begin{tabular}{ccccccc}
\hline & $Y_{1}$ & $Y_{2}$ & $Y_{3}$ & $Y_{4}$ & $Y_{5}$ & Rankings \\
\hline$\lambda=1$ & 0.3713 & 0.3787 & 0.2975 & 0.3205 & 0.2895 & $Y_{5} \succ Y_{3} \succ Y_{4} \succ Y_{1} \succ Y_{2}$ \\
$\lambda=2$ & 0.4451 & 0.4529 & 0.3523 & 0.4143 & 0.3660 & $Y_{3} \succ Y_{5} \succ Y_{4} \succ Y_{1} \succ Y_{2}$ \\
$\lambda=3$ & 0.5090 & 0.5053 & 0.3906 & 0.4889 & 0.4265 & $Y_{3} \succ Y_{5} \succ Y_{4} \succ Y_{2} \succ Y_{1}$ \\
$\lambda=4$ & 0.6558 & 0.5495 & 0.4211 & 0.5706 & 0.4758 & $Y_{3} \succ Y_{5} \succ Y_{2} \succ Y_{4} \succ Y_{1}$ \\
$\lambda=5$ & 0.5952 & 0.5776 & 0.4466 & 0.5892 & 0.5161 & $Y_{3} \succ Y_{5} \succ Y_{2} \succ Y_{4} \succ Y_{1}$ \\
\hline
\end{tabular}

Table 3 Results obtained by the generalized dual hesitant weighted Hausdorff distance.

\begin{tabular}{ccccccc}
\hline & $Y_{1}$ & $Y_{2}$ & $Y_{3}$ & $Y_{4}$ & $Y_{5}$ & Rankings \\
\hline$\lambda=1$ & 0.7800 & 0.7350 & 0.5300 & 0.6650 & 0.6200 & $Y_{3} \succ Y_{5} \succ Y_{4} \succ Y_{2} \succ Y_{1}$ \\
$\lambda=2$ & 0.7849 & 0.7440 & 0.5440 & 0.6953 & 0.6473 & $Y_{3} \succ Y_{5} \succ Y_{4} \succ Y_{1} \succ Y_{2}$ \\
$\lambda=3$ & 0.7898 & 0.7527 & 0.5572 & 0.7200 & 0.6691 & $Y_{3} \succ Y_{5} \succ Y_{4} \succ Y_{2} \succ Y_{1}$ \\
$\lambda=4$ & 0.7947 & 0.7611 & 0.5691 & 0.7395 & 0.6871 & $Y_{3} \succ Y_{5} \succ Y_{2} \succ Y_{4} \succ Y_{1}$ \\
$\lambda=5$ & 0.7995 & 0.7689 & 0.5797 & 0.7549 & 0.7026 & $Y_{3} \succ Y_{5} \succ Y_{2} \succ Y_{4} \succ Y_{1}$
\end{tabular}




\section{Summary}

In this paper, we have investigated the distance measures for DHFSs. Based on ideas of the well-known Hamming distance, we have developed a class of dual hesitant distance measures, and discussed their properties and relations as their parameters change. It should be pointed out that all of the above measures are based on the assumption that if the corresponding DHFEs in DHFSs do not have the same length, then the shorter one should be extended by adding the minimum value in it until both the DHFEs have the same length. In fact, we can extend the shorter DHFE by adding any value in it until it has the same length of the longer one according to the decision makers' preferences and actual situations.

\section{References}

[1] Bin Zhu, Zeshui Xu, Meimei Xia, Dual hesitant fuzzy sets, Journal of Applied Mathematics.

[2] L. A. Zadeh, Fuzzy Sets, Information and Control 8 (1965) 338-353.

[3] K. Atanassov, Intuitionistic fuzzy sets, Fuzzy Sets and Systems 20 (1986) 87-96.

[4] V. Torra, Hesitant fuzzy sets, International Journal of Intelligent Systems 25 (2010) 529-539.

[5] V. Torra, Y. Narukawa, On hesitant fuzzy sets and decision, in: The 18th IEEE International Conference on Fuzzy Systems, Jeju Island, Korea, 2009. pp. 1378-1382.

[6] Z.S. Xu, Deviation measures of linguistic preference relations in group decision making, Omega 17 (2005) 432-445.

[7] D.F. Li, C.T. Cheng, New similarity measures of intuitionistic fuzzy sets and application to pattern recognitions, Pattern Recognition Letter s 23 (2002) 221-225.

[8] W.L. Hung, M.S. Yang, Similarity measures of intuitionistic fuzzy sets based on Hausdorff distance, Pattern Recognition Letters 25 (2004) 1603-1611.

[9] P. Grzegorzewski, Distances between intuitionistic fuzzy sets and/or interval-valued fuzzy sets based on the Hausdorff metric, Fuzzy Sets and Systems 148 (2004) 319-328.

[10]Zeshui Xu, Meimei Xia, Distance measures for hesitant fuzzy sets, Information Sciences, 2011, 181 (11): 2128-2138.

[11]Zeshui Xu, Meimei Xia, On distance and correlation measures of hesitant fuzzy information, International Journal of Intelligent Systems, 2011, 26 (5): 410-425. 